\title{
Health Care Provider Processes Analysis
}

\author{
Lukasz Kawczynski and Marco Taisch \\ Department of Management, Economics and Industrial Engineering, \\ Politecnico di Milano, \\ Piazza L. da Vinci, 32 I-20133 Milano, Italy \\ \{lukasz.kawczynski, marco.taisch\}@polimi.it
}

\begin{abstract}
In every society there is a need for an efficient health care system. This is a case study paper that summarizes process analysis performed at a US provider clinic. This paper provides an analysis of factors (arrival accuracy and no shows) influencing main processes within the clinic. The numerical relations between influencing factors and key processes are exhibited. Moreover, the abilities of a health care provider to deal with variations of arrival time are exhibited. The predicted probabilities for arrival accuracy and no shows are discussed. The paper provides an interesting statistical approach to analyze operations of a health care provider, which can be beneficial for stakeholders of the clinic.
\end{abstract}

Keywords: Health care, Process analysis, Arrival time, No shows.

\section{Introduction}

Health care is an important aspect of every society. Recently, the subject itself is getting more attention as it is in need for change in many countries. Improvements have to be made in order to make it more efficient and patient driven. This is a case study paper describing part of the research conducted at a US nationwide health care provider, specializing in children care. This paper is part of larger research. Based on the study of outpatient clinic, the research aims to propose value definition and value chain model, which will be supported by simulation. This paper is analyzing flow of the patients through the clinic, aiming to show dependencies and relations between key processes, and how they are influenced by arrival times of patients. As a consequence, the ability of providers to deal with various scenarios are analyzed and the factors that could help predicting no shows and late arrival pointed.

\section{Literature Review}

Many authors were studying health care with focus on various aspects for inpatient and outpatient environment. The inpatient settings have received so far more attention in the literature than outpatient.

[10] analyzed length of stay (LoS) of patient in a hospital taking into consideration various data like: admission time, destination, discharge time, etc. The authors 
described and discussed couple of data models that could be used in connection with particular data warehouse environment. A transaction based model was proposed for facilitating LoS analysis and a snapshot-based for admissions, discharges and bed occupancy. [4] developed quality management model, which identifies problems, suggests solutions, develops a framework for implementation, and helps evaluate performance of inpatient health care services. [9] used industrial management principles to improve quality and efficiency of inpatient urgent care. Authors looked at urgent surgeries as an industrial process. General process analysis tools and process reengineering were used to develop the process. Process was described and measured to identify the bottlenecks. The care process was developed on the basis of the Deming cycle or the Plan-Do-Check-Act (PDCA) cycle. After implementation of suggested improvements authors found significant decrease in waiting times, rise in efficiency, and decrease of overtime hours. [2] proposed a flexible framework for modeling and simulation of inpatient health care. Authors offered a methodology which accommodates three types of view on health care system: process, resource, and organization. With a case study the use of the model is illustrated. [8] described a generalized net model of patient flows. The model aims to help improve management of the diagnostic consultative centers, through optimization of the use of resources. [6] proposed research a use of data mining and visualization techniques for decision support in planning and regional level management of Slovenian public health-care. They took attempt to identify areas which are atypical in terms of accessibility and availability. The authors contribute to theory by developing visualization methods that can be used to facilitate knowledge management and decision making processes. [3] presented a stochastic model of an individual patient's experience during a visit to a doctor's office. Authors apply semi Markov processes within outpatient clinic settings in order to model patient flow. The paper uses logical framework analysis (LFA), a matrix approach to project planning for managing quality. After applying in studied case, authors found improvements in quality performance of hospital. [1] were researching patient volume forecasting methods in walk-in outpatient clinics. On a large set of data they compared implementation of two forecasting models - autoregressive and growth curve of means. [5] described application of the discrete event simulation model to support analysis which led to improvement of outpatient procedure center processes Authors showed a bottleneck and as a result a need for additional resources. After reorganization of processes authors are examining 4 scenarios of different hedging strategies. The waiting times, efficiency, and utilization of an operation room are compared. Authors concluded with proposing an optimal design, i.e. being able to serve an assumed number of patients and at the same time minimizing waiting times and maximizing utilization. [7] concentrated on solving scheduling problems in ambulatory outpatient environment where patients are scheduled a few days in advance. With multi agent simulation researchers brought waiting times to constant level (little variation). Authors haven't been able to report significant decrease in waiting times.

Researchers have directed a lot of attention so far to the inpatient processes analysis. The outpatient clinic processes have received significantly less attention in the literature. 


\section{Methodology}

The methodology of this research could be divided into two parts. First part is responsible for picturing the processes and gathering the data. The process mapping activities were conducted in order to understand what processes are in the clinic. The data were gathered from the systems supporting every day operation of health care provider (patient management system).

The second part is data analysis aiming to show dependencies and relations between variables describing process performance. The analysis is divided into three stages. In the first one we want to show how arrival times are influencing operation. Regression models are built in STATA and relevant dependent variables screened. In the second step, we want to show the model for predicting time to appointment variable and no-shows. The regression models are constructed for each variable aiming to estimate prediction for early arrival for patients and prediction of no shows. In the final part, we want to determine how likely a clinic is able to cope with late arrivals and what are the consequences. The Markov chains are employed to study delayed arrivals and operational performance.

\section{Health Care Provider Description}

The health care provider taking part in this research is specialized in children care. The provider is operating through the chain of clinics all over east and south US. The studied clinic has 13 sub-clinics representing different specialties. The provider operates from 8 AM to 4 PM, Monday through Friday. The clinic serves on average 93 patients daily, which makes it a medium size health care provider.

\section{Processes at Health Care Provider}

The mapping activities revealed seven main groups of the processes, which are: preregistration, scheduling, eligibility verification (for insured patients), check-in, preexamination, medical examination, and billing. In this paper we are focusing on the processes within clinic (check-in, pre-examination, and medical examination). These processes are describing patient flow from arrival at the facility to check out.

The check-in process is including several sub processes. After patient arrival at the clinic, the sign in sub-process is performed during which patient information is verified and the system updated. This sub-process is followed by check-in, during which the final insurance verification is performed and co-pay (if any) collected. Patient is issued a buzz pager and is proceeding to a central waiting area.

The pre-examination process starts when patient's record is checked for any missing data. Afterwards, patient is called (via buzz pager) to pre-examination room. The reason for the visit is confirmed, appropriate vitals gathered and entered into the system.

The medical examination is started with a verification of allergies, medical reconciliation, medical and social history. Afterwards, the physical examination is performed. Doctor provides a diagnosis and updates patient record, entering any additional tests (if needed). After the visit is completed the nurse checks out patient. 


\section{Data description}

The system data count for $15000+$ records. During quality screening of system dataset we removed records that did not contain a complete data or contained an obvious error. After verification we received $4600+$ valid records. This data set is used for analyzing processes and arrival times. The dataset included variables as described in Table 1.

Table 1. Variables' means, standard deviations, and distributions

\begin{tabular}{llll}
\hline Variable & $\begin{array}{l}\text { Sample mean } \\
\text { (minutes) }\end{array}$ & $\begin{array}{l}\text { Standard deviation } \\
\text { (minutes) }\end{array}$ & Distribution \\
\hline Appointment time & 11,9 (hours) & 144,6 & Beta \\
Time to appointment & $-22,8$ & 31,2 & Normal \\
Arrival time & 11,48 (hours) & 141,6 & Beta \\
Check in time & 6,6 & 7,2 & Exponential \\
Waiting time & 25,2 & 24,6 & Weibull \\
Vitals time & 23,4 & 22,8 & Gamma \\
$\begin{array}{l}\text { Medical examination and check out } \\
\text { (including waiting time for provider) }\end{array}$ & 58,8 & 52,8 & Erlang
\end{tabular}

For analysis of no shows we used full dataset of 15000+ records, which describes an 8 month period of operation. More than 6500 records are no shows. Taking this into consideration no-shows seem to be an important problem in every day operation.

\section{Clinic Performance Influencing Factors}

We aim to determine what is influencing patient flow through the clinic by establishing links between variables describing main operational performance. The analysis is started with building statistical models in STATA linking medical examination length (the time that patient spends with a doctor including check out time and waiting time in exam room) and waiting time in lobby with other variables.

The linear regression model that is using medical examination time as a dependent variable showed with a p-value of zero that the variables that have influence are the ones that are describing late arrival of patient to the appointment and the time needed to gather vitals. Based on beta coefficients the most influence has a delay of arrival time. The regular coefficients indicate that for every 12 minutes of delay in patient arrival there is an hour increase in medical examination time (while other variables are hold constant). Naturally, this time is mostly consumed by waiting time, not the physical examination performed by a care provider. The variable describing time to gather the vitals suggest that there is negative correlation with medical examination $(-0,175)$. The longer time needed to gather the vitals the shorter medical examination time.

The Poisson regression model that is using waiting time as a dependent variable with a p-value of zero indicated that the variables influencing a waiting time are the ones that describe late arrival and the length of check in process. Every 10 minutes of late arrival is causing more than 12 minutes of additional waiting time in provider's 
lobby area (while other variables are hold constant). Moreover, the model suggests that every additional 5 minutes needed for check in would result in more than 2,5 minutes of additional waiting time (while other variables are hold constant).

The above statistical analysis is pointing out the dependencies between key processes performance and arrival times of patients.

\section{Prediction of Arrival Time and No Shows}

In order to analyze the probabilities of particular arrival times we created an additional binominal variable which classifies all the patients that arrived at least 5 minutes before an appointment as 1 (early arrival) and the rest as 0 (late arrival). The logistic regression model was built in STATA. The model indicated with a p-value of zero that there are two variables significant: appointment time and day of the week. Based on model, predicted probabilities suggested that patients that are having appointments early in the morning on Mondays on average are $7 \%$ less likely to arrive on time than patients having the same appointment on Friday. The difference is reduced to $4 \%$ for late afternoon appointments respectively. The probability of patient arriving at least 5 minutes before a scheduled appointment time is lower for first appointment in the morning (between 0,6 and 0,67 depending on a day of the week) than for the last one of the day (between 0,83 and 0,87 depending on a day of the week). Predicted probabilities of early arrival for a different appointment times are exhibited in Figure 1.

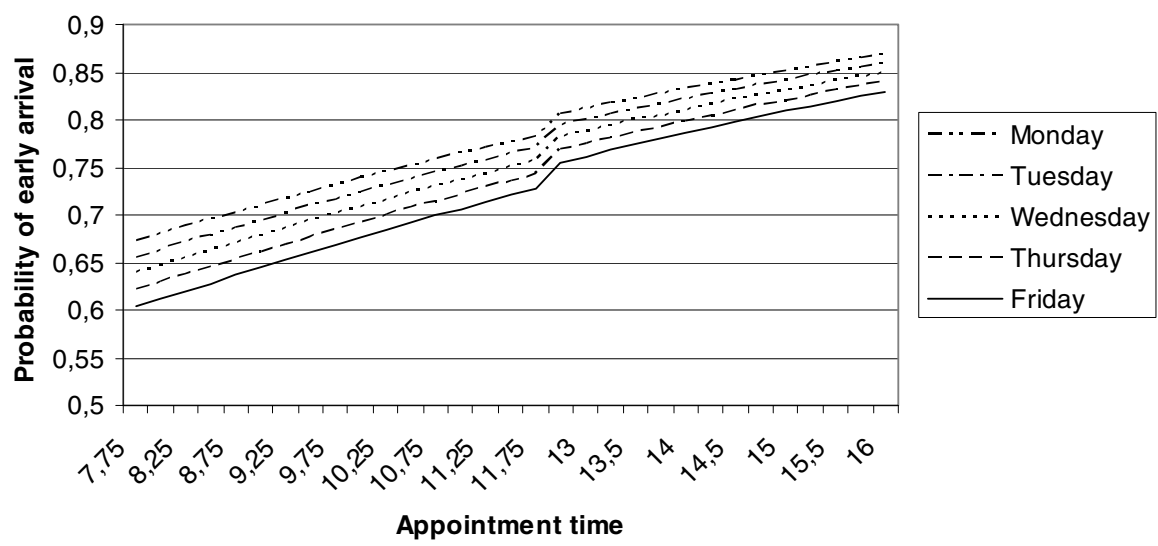

Fig. 1. Probability of early arrival as a function of appointment time

Moreover, within studied data period there is improvement in the patient early arrival time. During 8 months the early arrival probability increased by 0,1 .

In case of variable describing no shows, logistic regression was used. The variable has binominal values only. The model with a p-value zero suggest that variable describing day of the week and variable describing type of patient (new or returning) are significant only. The statistical model suggested based on predicted probabilities 
values that the probability of no show for a new patient is close to 0,46 , while for returning one only 0,37 . Taking into consideration the day of the week, there is higher chance of no show on Monday $(0,43)$ than on Friday $(0,4)$, and its constantly decreasing through the week.

\section{Process Implications of Late Arrivals}

The Markov chains are employed in order to determine how the clinic is able to deal with late arrivals and what the consequences are. The first step is the calculation of the matrix describing delays in arrival time and the difference between scheduled time and the actual time when patient was seen by care provider. In order to provide visibility, both variables are converted into interval variables. The implemented interval is 10 minutes, and the data is rounded down toward zero. The difference between arrival time and scheduled appointment time is taking into consideration only records that are having positive value, which means that patient had arrived late or in the best case right on time. Both variables have been limited by upper bound of 180 minutes, which is reasonable boundary for practical analysis. We built a 19 x 19 matrix where on one horizontal axis is an interval variable describing how long after scheduled appointment time patients arrive, while on vertical axis there is an interval variable which describes how many minutes after scheduled appointment time patient was seen by care provider. The matrix computes probabilities for each possible state based on the system data. The Markov chains are used to compute probabilities of patients being seen with certain delay by care provider. The process is repeated for patients arriving 10, 20, 30, 40, 50, 60, and 120 minutes past the scheduled appointment time. By multiplying subsequent matrixes, we receive probabilities for subsequent patients. The analysis is conducted for up to $K_{n+5}$ patient, where $K_{n}$ is a zero time patient. Below in Figure 2 and 3 are graphs that exhibit a cumulative probability and a delay in minutes counted from scheduled appointment time that patients is going to be seen by care provider. The analysis revealed that even a 10 minute delay is causing serious disturbances of the system. Patient arriving at the clinic 10 minutes past an appointment time has a $20 \%$ chance to be taken right away and $80 \%$ chance to be taken within 30 minutes from scheduled appointment time. The subsequent patients' probabilities are far lower. For the next arriving patient these probabilities are respectively less then $5 \%$ and less then $40 \%$. For third patients the situation is even worst. In figure 3 , the delayed arrival by 30 minutes is characterized by similar distribution function than the first case. Patient has less then $9 \%$ chance to be taken right away, and around $80 \%$ chance to be taken within 50 minutes from the scheduled appointment. In case of patients arriving after delayed one, they have less then $1 \%$ and $22 \%$ of chance respectively. Both graphs (for 10 and 30 minutes delay) are looking alike, and so are the plots for other delays' values. The difference might be notice in the angle of the rise. In case of relatively small delays this angle is pretty high, which means that clinic is likely to cope with this situation, while in case of large delays the angle becomes lower. 


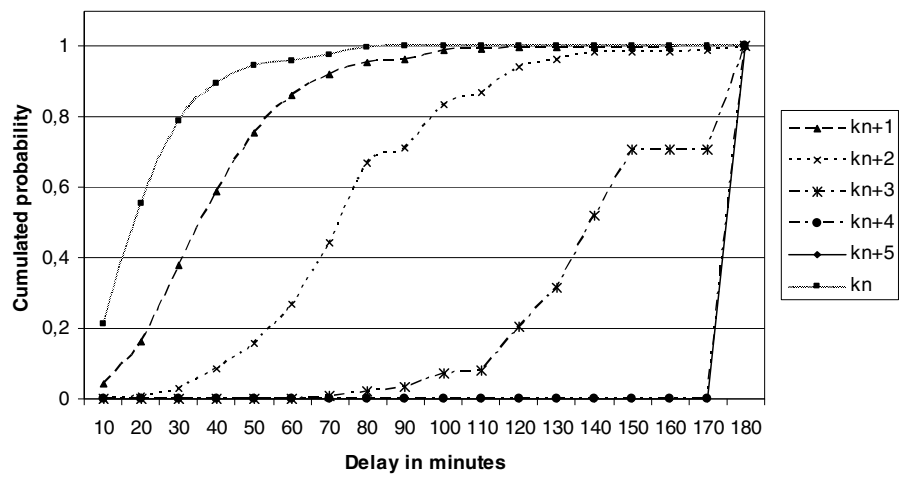

Fig. 2. Cumulative probability as a function of delay of patient being seen by care provider for patient arriving 10 minutes after an appointment time

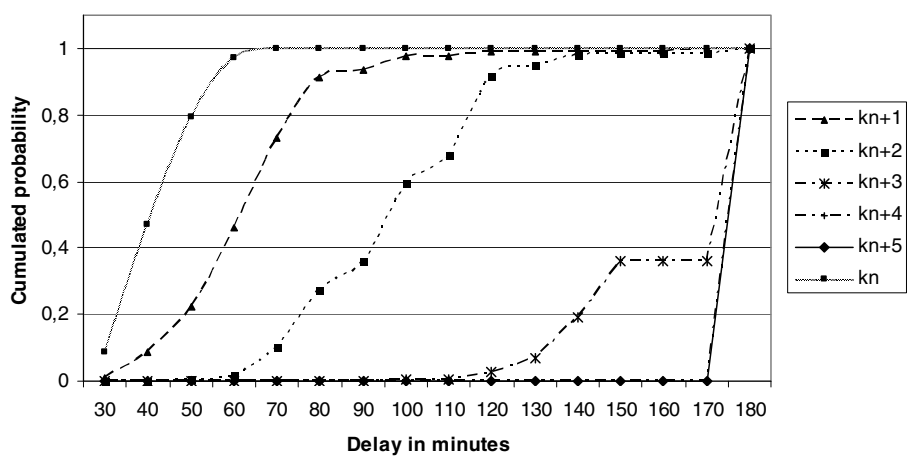

Fig. 3. Cumulative probability as a function of delay of patient being seen by care provider for patient arriving 30 minutes after an appointment time

\section{Conclusion and Further Research}

Health care is an important and at the same time difficult sector to manage. Many of the processes are sensitive and not all of them are manageable. The above analysis reveled how much the medical examination time and waiting times depends on patient accurate arrival. The analysis showed as well how the clinic is coping with late patients' arrivals. Based on above analysis, health care provider should implement tools encouraging patients to accurate arrival. Provider should emphasize to the patient while scheduling the appointment, how important is his/her arrival on time. It should be clear for patient that his/her on time arrival will minimize total time spent in the clinic. Moreover, provider should analyze the influence of various medical visit windows (the time in the system for one patient) on system sensitivity to late arrivals. This gap at studied case is established individually by each care provider, who is guided more by common sense than by hard data. 
Out of the above analysis the stakeholders and process owners should benefit by learning how the arrival time and no shows analysis could be conducted, and how they influence every day operation.

Further research should be done in order to study the relation between arrival time and actual waiting time for a provider and a time that patient spends with provider, as these variables have been treated here jointly. Moreover, in the future more health care providers should be studied in order to propose valid generalized results, applicable to any provider.

\section{References}

1. Abdel-Aal, R.E., Mangoud, A.M.: Modeling and Forecasting Monthly Patient Volume at a Primary Health Care Clinic Using Univariate Time-Series Analysis. Computer Methods and Programs in Biomedicine 56, 235-247 (1998)

2. Augusto, V., Xie, X., Grimaud, F.: A Framework for the Modeling and Simulation of Health Care Systems. In: Proceedings of the 3rd Annual IEEE Conference on Automation Science and Engineering (2007)

3. Cote, M.J., Stein, W.E.: A Stochastic Model For a Visit to the Doctor's Office. Mathematical and Computer Modelling 45, 309-323 (2007)

4. Dey, P.K., Hariharan, S.: Integrated approach to healthcare quality management: a case study. The TQM Magazine 18(6), 583-605 (2006)

5. Huschka, T.R., Denton, B.T., Narr, B.J., Thompson, A.C.: Using Simulation in the Implementation of an Outpatient Procedure Center. In: Proceedings of the 2008 Winter Simulation Conference (2008)

6. Lavrac, N., Bohanec, M., Pur, A., Cestnik, B., Debeljak, M., Kobler, Andrej: Data Mining and Visualization for Decision Support and Modeling of Public Health-Care Resources. Journal of Biomedical Informatics 40, 438-447 (2007)

7. Stiglic, G., Kokol, P.: Patient and Staff Scheduling Multi-Agent System. In: IEEE 3rd International Conference on Computational Cybernetics - Proceedings, vol. 2005, pp. 25-28 (2005)

8. Tasseva, V., Peneva, D., Atanassov, K., El-Darzi, E., Chountas, P., Vasilakis, C.: Generalized Net Model for Outpatient Care in Bulgaria. In: Twentieth IEEE International Symposium on Computer-Based Medical Systems, CBMS 2007 (2007), 0-7695-2905-4/07

9. Torkki, P.M., Alho, A.I., Peltokorpi, A.V., Torkki, M.I., Kallio, P.E.: Managing urgent surgery as a process: Case study of a trauma center. International Journal of Technology Assessment in Health Care 2, 255-260 (2006)

10. Vasilakis, C., El-Darzi, E., Chountas, P.: An OLAP-Enabled Software Environment for Modeling Patient Flow. In: 3rd International IEEE Conference Intelligent Systems (September 2006) 